\title{
Two Design Principles for the Design of Demonstrations to Enhance Structure-Property Reasoning
}

\author{
Marie-Jetta den Otter ${ }^{1, * \mathbb{C}}$, Michiel Dam ${ }^{1}$, Ludo B. F. Juurlink ${ }^{2} \mathbb{D}$ and Fred Janssen ${ }^{1}$ \\ 1 Leiden University Graduate School of Teaching (ICLON), Leiden University, P.O. Box 905, \\ 2300 AX Leiden, The Netherlands; m.dam@iclon.leidenuniv.nl (M.D.); fjanssen@iclon.leidenuniv.nl (F.J.) \\ 2 Leiden Institute of Chemistry (LIC), Leiden University, P.O. Box 9502, 2300 RA Leiden, The Netherlands; \\ 1.juurlink@chem.leidenuniv.nl \\ * Correspondence: m.den.otter@iclon.leidenuniv.nl
}

check for updates

Citation: den Otter, M.-J.; Dam, M.; Juurlink, L.B.F.; Janssen, F. Two Design Principles for the Design of Demonstrations to Enhance Structure-Property Reasoning. Educ. Sci. 2021, 11, 504. https://doi.org/ 10.3390/educsci11090504

Academic Editor: Gavin T. L. Brown

Received: 6 July 2021

Accepted: 29 August 2021

Published: 4 September 2021

Publisher's Note: MDPI stays neutral with regard to jurisdictional claims in published maps and institutional affiliations.

Copyright: (c) 2021 by the authors. Licensee MDPI, Basel, Switzerland. This article is an open access article distributed under the terms and conditions of the Creative Commons Attribution (CC BY) license (https:// creativecommons.org/licenses/by/ $4.0 /)$.

\begin{abstract}
Structure-property reasoning (SPR) is one of the most important aims of chemistry education but is seldom explicitly taught, and students find structure-property reasoning difficult. This study assessed two design principles for the development of structure-property reasoning in the context of demonstrations: (1) use of a POE task (predict-observe-explain) and (2) use of the domain-specific particle perspective, both to increase student engagement and to scaffold micro-level modeling. The aim of the demonstration series was to teach structure-property reasoning more explicitly to pre-university students (aged 15-16). Demonstrations pertained to the properties of metals, salts and molecular compounds. The SPR instrument was used as a pretest and posttest in order to gain insight into the effects on structure-property reasoning. In addition, one student (Sally) was followed closely to see how her structure-property reasoning evolved throughout the demonstrations. Results show that after the demonstrations students were more aware of the structure models at the micro-level. The students also knew and understood more chemical concepts needed for structure-property reasoning. Sally's qualitative data additionally showed how she made interesting progress in modeling micro-level chemical structures. As we used conventional demonstrations as a starting point for design, this could well serve as a practical tool for teachers to redesign their existing demonstrations.
\end{abstract}

Keywords: chemistry education; structure-property reasoning; demonstrations; POE task; particle perspective

\section{Introduction}

In chemistry, structure-property reasoning is considered to be one of the most important overarching constructs [1]. It is a type of chemical reasoning in which chemists explain the macroscopic properties of a compound in terms of the structure level of this type of compound, namely the particles, their organization and interactions. The properties refer to observable properties of compounds such as melting point, hardness and solubility. Structure-property reasoning is important for explaining and predicting properties of compounds. It is also critical for designing new compounds with desired properties. Therefore, this type of reasoning takes a prominent place in various curricula over the world including in the Netherlands $[2,3]$.

Chemistry students generally have problems developing such reasoning resulting from rather particular difficulties. The first difficulty is the requirement to switch between different levels of thought within chemistry [4]. When observable phenomena need to be explained and interpreted at the micro-level, students have to connect the two levels using models of particles and their interactions. However, as students are mostly novices in structure-property reasoning, they tend to stick with the macro-level observations and simply use former experiences to explain the properties instead of using the microlevel models [5]. Reasons for this are being unfamiliar with the micro-level models and 
experiencing difficulties with their interpretation, e.g., precisely how micro-level particles interact to account for the observed properties at the macro-level.

The second difficulty in developing structure-property reasoning is that micro-level particles cannot be seen with the naked eye or even with the best optical microscope. Consequently, structure-property reasoning becomes rather abstract and students draw on more general problem-solving skills to solve chemical problems instead of on a deep understanding of structure-property relationships $[5,6]$.

Difficulties in structure-property reasoning may primarily be regarded as a consequence of how chemistry is taught and how chemistry curricula are organized. As most national curricula are organized around chemical topics (chemical bonding, etc.) instead of explicit conceptual relationships or cross-cutting forms of chemical thinking, teachers are not facilitated to explicitly teach structure-property reasoning. As a result, students develop heuristics such as "surface similarity" (compounds with similar appearances are compounds of the same group and thus they have the same properties) [7] to answer questions in this realm. However, their understanding of these structure-property relations remains poor [7-9].

Literature suggests that the teaching of structure-property reasoning should be explicit and centered on the "core idea" of structure-property relationships $[1,10]$. Students should learn to connect the real with the modeled world and to use structure models to explain real chemical phenomena [1].

The use of demonstrations to show chemical phenomena has been suggested as a teaching practice to explicitly teach structure-property reasoning [11-13]. In conventional demonstrations, learning starts at a macro-level familiar to most students. Teachers demonstrate real chemical phenomena, and students are expected to observe what happens at the macro-level before the teachers provide a micro-level explanation for the chemical phenomenon at hand (observe-explain demonstration) [14].

What is lacking in most of the typical observe-explain demonstrations is that: (1) teachers do not let their students activate prior knowledge to build on what they already know and (2) most teachers do not ask students to model micro-level explanations themselves [14]. These two imperfections of a conventional demonstration lead to a twofold need: an approach in which students can actively build on what they already know and a means to stimulate and guide micro-level modeling by students in connection with a demonstration. The former may be done by predicting outcomes prior to performing a demonstration. For the latter, an explicit scaffold for students' micro-level modeling may be introduced [6].

In the study presented in this article, we designed and tested a demonstration-based lesson series aimed at improving structure-property reasoning. For the design of the lesson series, we explicitly used conventional demonstrations as a basis and applied two design principles: (1) the introduction of a POE task (predict-observe-explain) to demonstrations [13,15-17] to stimulate students' engagement and their modeling process and (2) scaffolding of the POE task with a domain-specific particle perspective $[18,19]$ in order to explicitly guide the modeling at the micro-level for students in the "explain" phase of the POE task. This particle perspective consisted of a question agenda with questions on which type of compound, which properties and which type of particles. Next, the demonstration-based lesson series was tested for the level of students' structure-property reasoning as reproduction, understanding, application and evaluation. We studied student engagement as they developed models for the structure level for three dominant types of chemical compounds: metals, salts and molecular compounds. The learning objective for the students in the upper pre-university tier of secondary education was to acquire these structure models. We performed the lesson series and investigated how students reproduced, understood, applied and evaluated structure-property reasoning. 


\section{Theoretical Framework}

To improve students' structure-property reasoning, it should be taught explicitly and be in line with students' macroscopic orientation [5]. This can be achieved by using real chemical phenomena in which properties of substances are investigated [1]. Students can be effectively engaged with such real-life chemical phenomena by using demonstration experiments [20]. Due to the way many teachers incorporate the demonstration into their teaching practice, learning efficiency for the students is low [21]. Although the students are engaged by questions about what they have just observed, they are given few opportunities to discover for themselves how to explain the chemistry phenomena using the structurelevel models, let alone to discover and create these models themselves. As a result, the demonstration is little more than a beautiful show and learning efficiency remains low [21].

The question arises: to what extent teachers are able to offer students opportunities to think for themselves during a demonstration? How can students be actively and explicitly engaged in structure-property reasoning? Teaching practices that use conventional demonstrations are often characterized by teachers presenting theory before the demonstration, thereby reducing students' explicit engagement with structure-property reasoning. Consequently, students passively observe the demonstration and opportunities for active learning are missed.

To overcome these problems described in the paragraph above, an active role for the student is necessary [22]. Our first design principle, the addition of a POE task (predictobserve-explain) to conventional demonstrations $[13,15-17]$ was intended to achieve this. In a POE task, students are challenged to learn actively by predicting the outcome of a demonstration and justifying their predictions. Next, they describe what they observe during the demonstration, and, afterward, they explain their observations and reconcile any discrepancy between their predictions and observations. This technique has been frequently investigated over the years $[15,16,22-32]$. Therefore, it is a known approach in science education research, but the implementation is lacking. Besides fostering engagement, the POE task can be used to reduce misconceptions $[29,30]$ and it can help students to improve their learning outcomes [31]. The POE task is also suitable for enabling students to model the structure level of a compound in order to explain a certain property [33]. Finally, it encourages students to engage in explicit structure-property reasoning by connecting their macroscopic observations to their models of the structure level [32]. We believe that these characteristics of the POE task, such as students' active engagement and the opportunities for students to model themselves, give the POE task potential to reinforce demonstrations and improve students' structure-property reasoning.

Adding a POE task changes the order of teaching activities for demonstrations (Figure 1). A conventional demonstration has three steps: introduction or orientation, show and observe and explain. In all three steps, the teacher takes the lead. Even in the observation step, teachers direct the students' attention to important observations (and distract them from undesired observations).

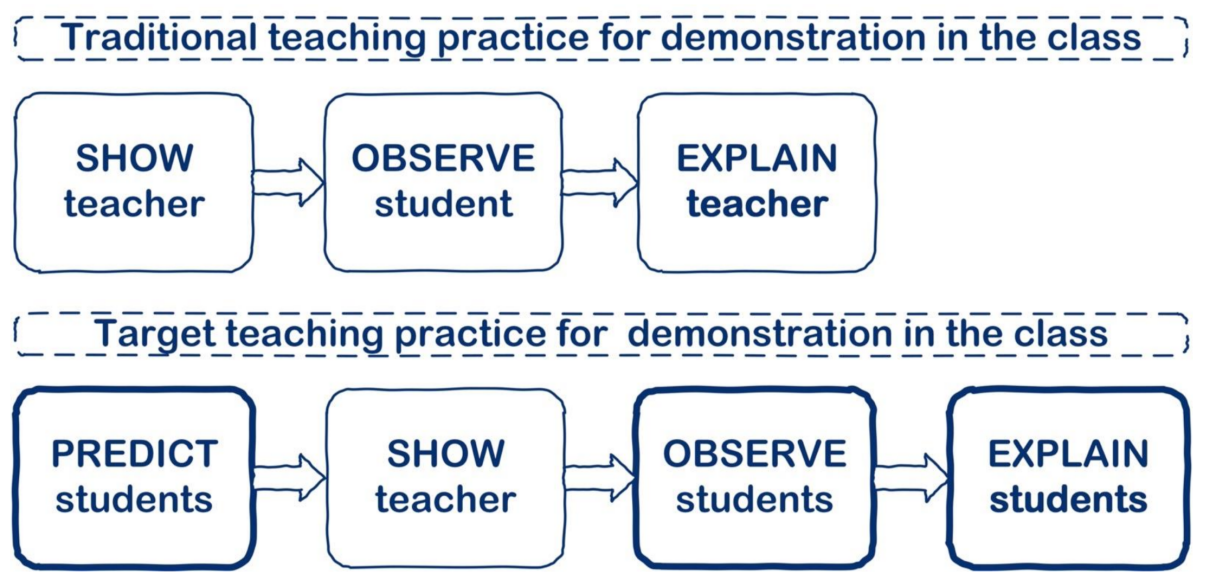

Figure 1. Conventional and target practice for demonstration in the class. 
The target teaching practice of a POE-task-based demonstration consists of an extra step "predict". In addition, all the steps (except for "show") have become student-centered to get students more engaged with the demonstration. In this way, students are challenged to reason using micro-level structures to explain the demonstrated properties on the macrolevel. For this, they can create and use micro-level structure models.

When students start creating the micro-models in the "explain" step of the POE task, they need to know the conditions for a micro-macro explanation. The students need insight into the underlying structure of the explanation and the corresponding questions that can be asked to systematically address the problem. A scaffold can be of assistance [34]. Hence, we introduced our second design principle: scaffolding of the POE task with a domain-specific particle perspective. [18,19]

As illustrated in Figure 2, this particle perspective consists of a set of questions (a question agenda) that experts in chemistry would unconsciously ask themselves when dealing with structure-property relations $[18,19]$. For example, when dealing with a problem about the potential solubility of poly-4-hydroxystyrene in a basic solution, one needs to know-besides which substance and which property-which type of particles is involved (polymer with hydroxy groups and basic particles) and which type of bonds (ion-dipole bonds) plays a role.

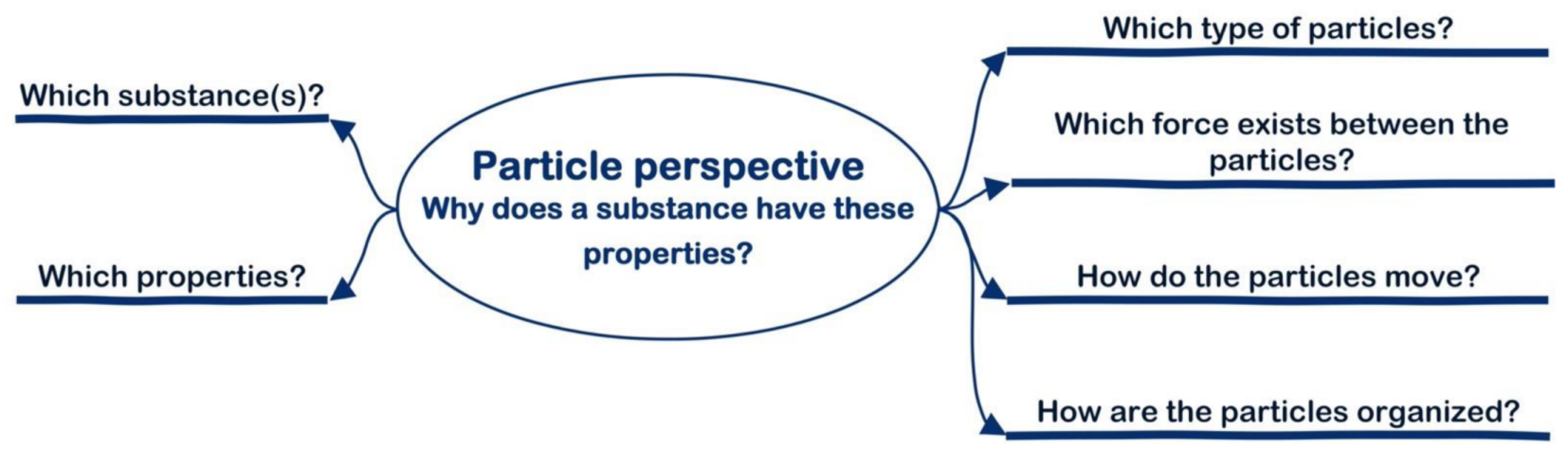

Figure 2. Question agenda of the particle perspective.

Relative novices, as students mostly are, can use the question agenda to interrogate the problem. In this way, the particle perspective can act as a scaffold for the students to model the structure models of the micro-level, which in turn can be used for explaining the macrolevel. Students need to get acquainted with the questions and the answers, i.e., chemical concepts of this particle perspective, to become proficient in structure-property reasoning. In addition, the questions and answers can be seen as thinking tools for structure-property reasoning. Figure 3 shows an elaborated version of the particle perspective with all the chemical concepts needed for structure-property reasoning explored in this study.

In the "explain" phase of the POE task, students need to explain their observations. The particle perspective can therefore act as a scaffold to facilitate the students in this phase. By answering the questions of the perspective, the students are scaffolded to the appropriate chemical concepts needed to explain their observations.

The domain-specific perspectives can also act as a stepping stone to expand the required chemical concepts needed for structure-property reasoning. When used repeatedly in multiple settings such as lessons, new chemical concepts can be added to the question agenda, and questions can be divided into several sub-questions. As students' knowledge grows, the particle perspective grows, and more complex problems can be investigated. Furthermore, the students' knowledge will be organized by the particle perspective. This gives students an overview, and interdependences become clear [18,19].

The two design principles served as the basis for our design study. We aimed to design demonstrations with a POE task and the scaffold of the particle perspective in order to en- 
gage pre-university students in modeling the structure level of metals, salts and molecular compounds and to enable them to learn how to perform structure-property reasoning.

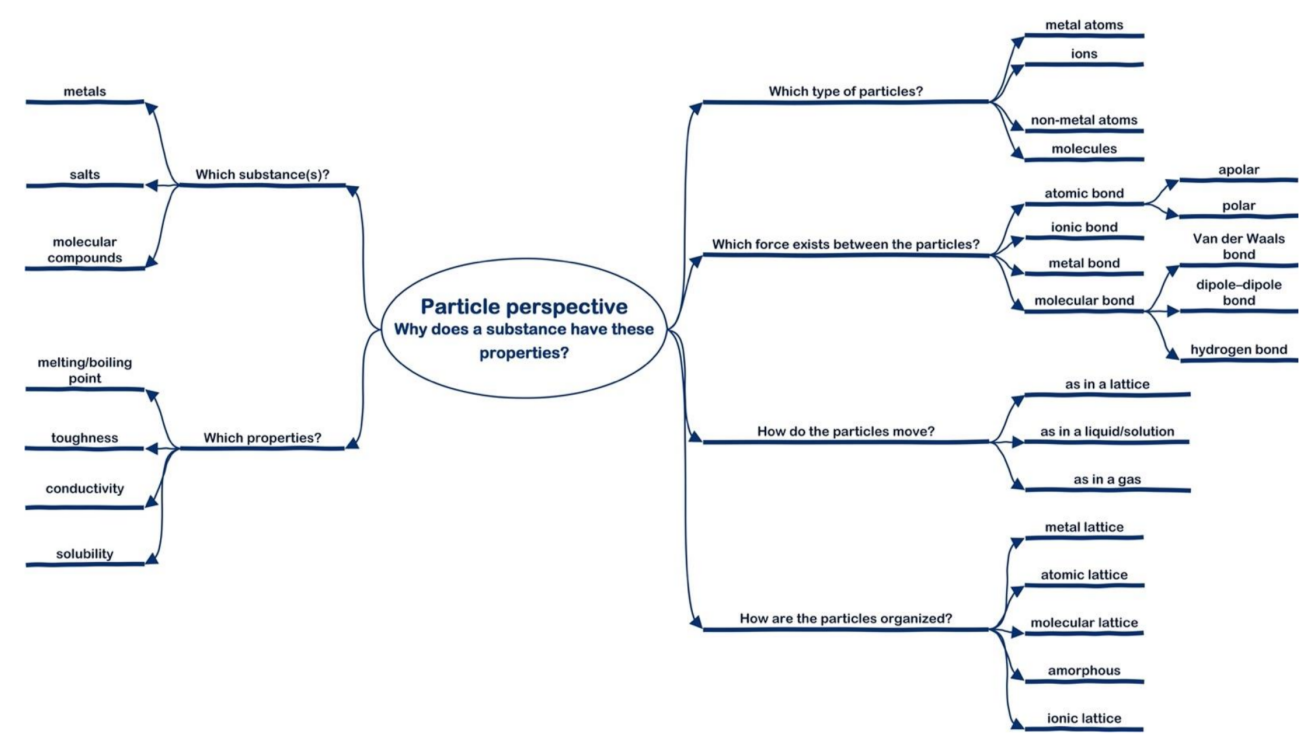

Figure 3. Particle perspective elaborated with the chemical concepts a student should master in the fifth year of the pre-university track.

\section{Materials and Methods}

\subsection{Research Design}

Using a one-group pretest-posttest design, the effectiveness of a demonstration-based lesson series with a POE task and the particle perspective as scaffold was investigated. The aim of the demonstration-based lesson series was to stimulate and develop students structure-property reasoning. To be more specific, the students had to learn the chemical concepts, e.g., hydrogen bridge or ions, that are associated with the micro-models of metals, salts and molecular compounds. Figure 3 shows all the chemical concepts offered in these demonstrations and thus the learning objectives for the students. They also had to construct and apply the micro-models themselves with the associated chemical concepts.

The activities were designed by the first author and piloted in her own teaching practice. This pilot showed that the selected design worked well for the group metals and the group salts. For molecular compounds, however, we noticed that students generally were not able to predict properties. Consequently, we redesigned the lesson on molecular compounds (see "Overview of the Lesson Series" below). The adapted lessons were again provided by the first author.

\subsection{Setting and Participants}

The lesson series was performed in two cohorts in a Dutch secondary school: cohort 18-19 and cohort 19-20. Table 1 shows the number, gender and ages of students in both cohorts.

Table 1. Composition of the two cohorts.

\begin{tabular}{lccccc}
\hline & Year & Students & Male & Female & Age \\
\hline Cohort 18-19 & 4 & 37 & 21 & 16 & $15-16$ \\
Cohort 19-20 & 4 & 16 & 9 & 7 & $15-16$ \\
\hline
\end{tabular}

The students were in the fourth year of the pre-university track. In the third year, they had been introduced to chemistry with an introduction to the topics: substances and their properties, particle models, separation methods, chemical reactions, atoms, molecules, 
metals, organic compounds, reaction heat, reaction rate, stoichiometry, fuels and plastics. Our designed demonstration lessons were part of a topic about chemical bonding. Before this course, the students had learned about Bohr's atomic model, mole, stoichiometry and concentration.

\subsection{Overview of the Lesson Series}

The two design principles were incorporated into the lesson series which comprised three lessons of $50 \mathrm{~min}$ each. Properties of the three types of substances (metals, salts and molecular compounds) were demonstrated. Students then engaged in activities to discover the structure models underlying some of the common properties of these three types of compounds (Figure 4).

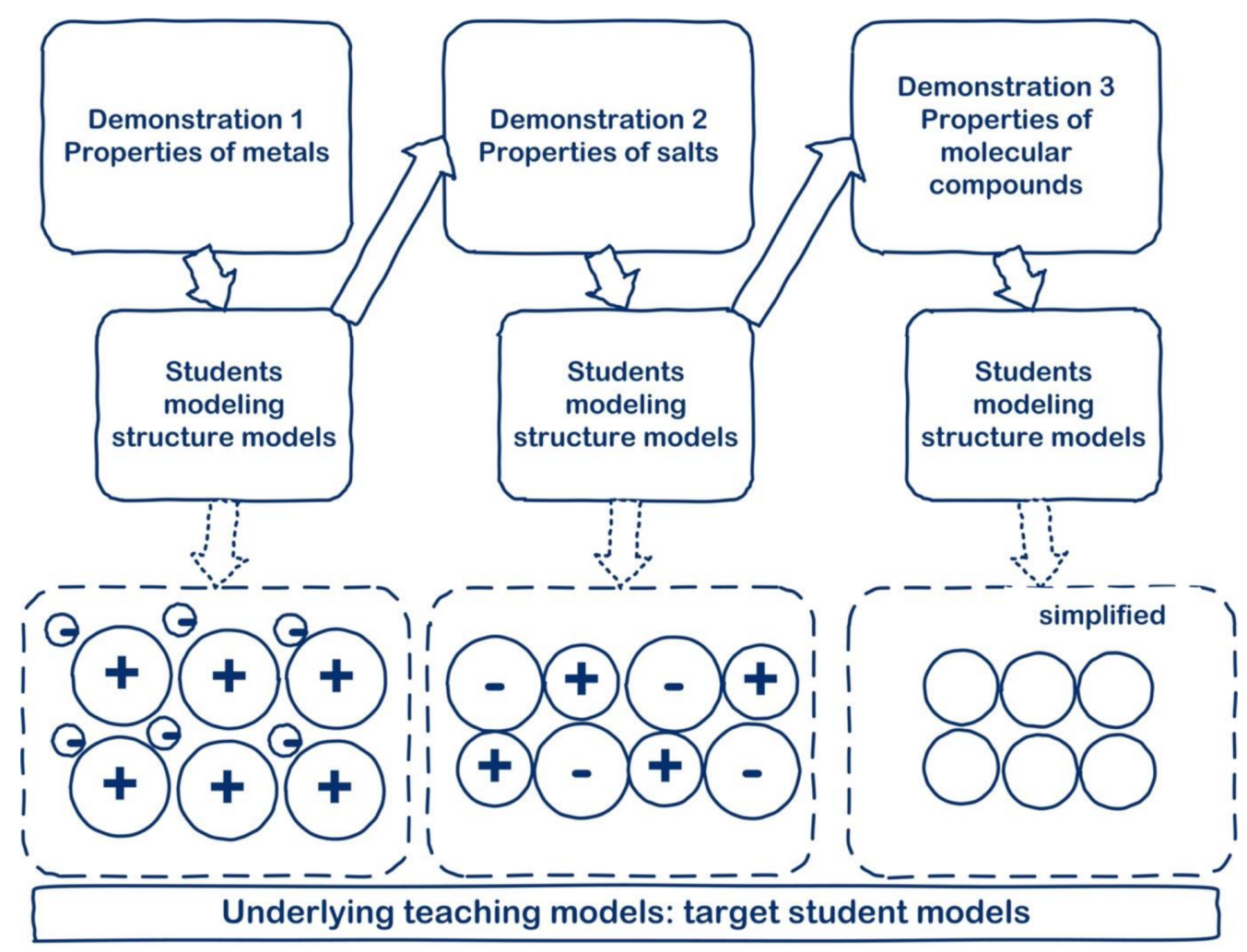

Figure 4. Overview of the lesson series.

\section{STEP 1: PREDICT}

The demonstration lesson series started with metals. Several metals were displayed on the teacher's desk, such as iron, copper, lead, aluminum and zinc. First, the question agenda of the particle perspective was handed out to the students, and the teacher asked them to complete this with answers suitable for the metals (in order to obtain their prior knowledge). Next, the students were asked to predict the properties of this group of substances $(\mathrm{P}=$ predict $)$. These predictions of the properties were collected in a class discussion.

\section{STEP 2: OBSERVE}

Properties shown in this step were: general aspects such as color, phase at room temperature, malleability, hardness and electrical conductivity. During the demonstration, there was a class discussion solely about the macroscopic properties of the metals. The order of the shown properties was chosen to facilitate a step-by-step development to build on what students had already studied in their third year (a more general particle model) toward a more sophisticated structure model of metals, the learning objective for this course. The students had to observe the demonstrated properties of each of these metals.

STEP 3: EXPLAIN 
In this step, the students were asked to produce a structure model of the shown substance based on their observations of the properties. They could use the question agenda from the particle perspective as a scaffold (Figure 1), and they discussed their models in small groups. After that, their structure models were discussed in a whole-class discussion to enable the students to test their own structure models. After approximately three iterations of the first student-generated models, their models were compared with the commonly accepted teaching models.

The students were asked to create a structure model for the metals that explained the properties shown in the demonstration. After the group discussions, the teacher discussed the common denominator of the student-generated structure models. Then, the teacher asked the students to show how the property malleability could be explained in the structure model. After group discussions, this was again discussed in the whole class. After that, the teacher asked the students to produce two iterations: (1) to adapt the structure model to explain the hardness of alloys and (2) to adapt the structure model to explain conductivity of electricity. Finally, students supplemented the question agenda of the particle perspective from the beginning with the discovered concepts and structure models.

More information about the demonstration lesson series can be found in the Appendix A supplementary information, where the demonstration protocols for the metals, salts and molecular compounds are described (See Appendix A).

Based on the pilot, we used a slightly different approach for the molecular compounds. Students found it difficult to predict the various properties of the molecular compounds. Consequently, they found modeling of the underlying structures to be complicated, and thus less complex structure models were needed to enable a constructive modeling process. For this reason, we simplified the structure model of the molecular compounds by concentrating on the general molecular interaction. In later lessons, this simplified model was explored by giving instructions about the various types of molecular interactions. In addition, it appeared the students needed an explicit scaffold for the modeling process. To offer this, we used the question agenda of the particle perspective to structure the demonstrations.

For these reasons, we adapted the structure of the demonstration lesson as follows: the lesson started with the questions of the particle perspective, and the class was asked to give suggestions on which property should be demonstrated by the teacher for discovering the answer to those questions (P phase). For example, to discover the type of particles, students could argue that conductivity should be demonstrated to reveal whether the particles were charged. Subsequently, all necessary properties, such as boiling point and conductivity, were demonstrated ( $\mathrm{O}$ phase) by the teacher, and the questions of the particle perspective concerning the micro-level were discussed together with the associated chemical concepts (E phase). At the end of the demonstration for the molecular compounds, the students completed the particle perspective again. An additional advantage of this approach was that it gave students the opportunity to explicitly practice the questions of the particle perspective.

\subsection{Data Collection and Data Analysis}

We gathered several types of data about the level of proficiency in structure-property reasoning using a combination of quantitative and qualitative instruments. We gathered data using the SPR instrument (structure-property reasoning instrument) [35], which was developed in previous research in order to estimate various aspects of structure-property reasoning at different levels of mastery. To provide insight into how the demonstrationbased lesson series impacted learning, we also gathered data in the form of manifest student products (student structure models, perspectives) and audio recordings of student group discussions.

The SPR instrument was administered as a pretest and posttest for both cohorts. Student results on the pre- and posttests were compared statistically to determine significant growth in structure-property reasoning. The SPR instrument consists of four tasks (see 
Table 2): an unframed and framed sorting task and an unframed and framed mapping task, all four based on the particle perspective.

Table 2. Description of SPR instrument.

\begin{tabular}{|c|c|c|c|}
\hline $\begin{array}{c}\text { Order of } \\
\text { Performance }\end{array}$ & Task & Description & Instruction \\
\hline 1 & $\begin{array}{l}\text { Unframed } \\
\text { sorting task }\end{array}$ & $\begin{array}{l}16 \text { problems on cards, all containing a structure } \\
\text { aspect and a property aspect, have to be sorted } \\
\text { into groups. Each group should be given a name. }\end{array}$ & $\begin{array}{c}\text { You received } 16 \text { cards with problems. Sort these cards } \\
\text { in groups based on } \\
\text { underlying common chemical concept. } \\
\text { Give each group an appropriate name. Form at least } 2 \\
\text { groups and maximal } 15 \text { groups. }\end{array}$ \\
\hline 2 & $\begin{array}{c}\text { Framed } \\
\text { sorting task }\end{array}$ & $\begin{array}{c}16 \text { problems on cards, same as unframed, have to } \\
\text { be sorted into four groups, namely } \\
\text { molecular/bonding, molecular/lattice, ionic, } \\
\text { metallic. }\end{array}$ & $\begin{array}{c}\text { Shuffle your } 16 \text { cards and sort them } \\
\text { in the four groups as stated on your worksheet: } \\
\text { molecular/bonding, } \\
\text { molecular/lattice, ionic, metallic. } \\
\text { Every group should contain at least one card. } \\
\text { In front of you, you see the questions of the particle }\end{array}$ \\
\hline 3 & $\begin{array}{l}\text { Unframed } \\
\text { mapping task }\end{array}$ & $\begin{array}{c}\text { Participants receive questions of } \\
\text { particle perspective (see Figure 2). The questions } \\
\text { should be completed by } \\
\text { answers in form of chemical concepts. Creating } \\
\text { hierarchy is allowed. }\end{array}$ & $\begin{array}{l}\text { perspective. } \\
\text { A perspective is a way of questioning your topic or } \\
\text { problem. } \\
\text { Complete the questions with the } \\
\text { appropriate chemical concepts. } \\
\text { You are allowed to form a hierarchy. }\end{array}$ \\
\hline 4 & $\begin{array}{c}\text { Framed } \\
\text { mapping task }\end{array}$ & $\begin{array}{l}\text { Participants receive questions of } \\
\text { particle perspective (see Figure 2) and } 30 \text { chemical } \\
\text { concepts. The concepts should be placed under the } \\
\text { appropriate question. } \\
\text { Creating hierarchy is allowed. }\end{array}$ & $\begin{array}{l}\text { Again, you are given the questions of } \\
\text { the particle perspective. } \\
\text { Complete the questions with } \\
\text { the given } 30 \text { chemical concepts. } \\
\text { You are allowed to form a hierarchy. }\end{array}$ \\
\hline
\end{tabular}

In the sorting tasks, the percent pairs $(\% \mathrm{P})$ for the structure level and the property level were determined. The percentages of pairs of cards equal to pairs found in the ideal structure or property sort formed by the participant were determined. The more similar a sort is to an ideal sort-either structure or property-the higher the percent pairs will be.

To provide insight into the type of group names the students used to categorize the formed groups in the unframed sorting task, the group names were coded by type of category name with the codes "referring to structure", "referring to property" or "other", as shown in Table 3. In the framed sorting task, the framed difference (FD) score was determined. The FD score is defined by the number of cards that are placed in a group other than the ideal sort.

Table 3. Coding scheme.

\begin{tabular}{cc}
\hline Codes & Example of Group Names \\
\hline Referring to structure & Electrons, hydrogen bonds, atomic bond, lattice \\
Referring to property & Hardness, density, conductivity, phase, solubility \\
Other & Polymers ... \\
\hline
\end{tabular}

In the unframed mapping, the number of given chemical concepts was counted and judged on correctness. The given chemical concepts were compared to the reference map (Figure 3). In the framed mapping task, the percentage of correctly placed chemical concepts was determined.

We also gathered qualitative data. These consisted of manifest student products (student structure models, perspectives) and audio recordings of student group discussions. In order to gain insight into how the demonstration-based lesson series impacted student learning, we chose to present a case study in which we described how one student's learning (Sally, cohort 19-20) was impacted by the lesson series. Sally (a fictive name) was chosen because her learning progression was a clear example of how students developed during the demonstration lessons. This student, Sally, collaborated with three female students in one group. We collected Sally's structure models about the topic of the group 
metals (first demonstration) and audiotaped Sally's group discussions. Resulting drawings and group discussions were analyzed as follows. First, we transcribed Sally's group discussions around the topic of metals and compared these to the drawings that Sally made in order to map how the modeling process of the structure level (for metals) progressed. Next, we analyzed in these transcripts how Sally's group spoke about the properties of malleability, hardness and electrical conductivity and how these properties were visible in their structure models. The main starting question for analysis was how the group adapted their first structure model of metals and how they progressed to the final structure model of metals.

\section{Results}

In this section, we first present the quantitative SPR instrument outcomes. Next, we present the case study of Sally.

\subsection{Pre- and Posttest by the SPR Instrument}

\subsubsection{Framed Mapping Task}

The framed mapping task tested whether the students acquired the offered chemical concepts of the particle perspective. Table 4 shows that the number of correctly placed chemical concepts increased significantly in the posttest. Students barely made any mistakes after the demonstration series. This result shows that students acquired and understood the chemical concepts needed for structure-property reasoning. The students were able to connect the chemical concepts with the corresponding question of the particle perspective.

Table 4. Results of the framed mapping task.

\begin{tabular}{cccc}
\hline \multirow{3}{*}{ Number of correct answers } & Cohort & Pre & Post \\
\hline \multirow{2}{*}{ Percentage } & Cohort 18-19 & 27 & 33 \\
& Cohort 19-20 & 31 & 35 \\
& Cohort 18-19 & $74 \%$ & $93 \%$ \\
& Cohort 19-20 & $86 \%$ & $97 \%$ \\
\hline
\end{tabular}

\subsubsection{Unframed Mapping Task}

Table 5 shows that the perspective maps created by the students were more comprehensive in the posttest compared to the pretest. The students provided more answers, i.e., chemical concepts, and their mapping was more comparable to the reference map. The results show that students were able to reproduce the learned concepts. Furthermore, they understood and applied the learned chemical concepts by connecting them to the corresponding question.

Table 5. Results of the unframed mapping task.

\begin{tabular}{ccccc}
\hline Number of & Cohort & Pre & Post & Difference \\
\hline \multirow{2}{*}{ Answers } & Cohort 18-19 & 14 & 20 & +6 \\
Answers comparable & Cohort 19-20 & 16 & 26 & +10 \\
with reference map & Cohort 18-19 & 6 & 15 & +9 \\
\hline
\end{tabular}

\subsubsection{Framed Sorting Task}

The demonstration lesson contributed to greater proficiency in structure-property reasoning. Both cohorts showed an increase in the \%P-structure score in the posttest. Students' sorts in the predetermined categories (framed mapping task) were more similar to the ideal structure sort. This probably means that after the demonstrations, students were more able to apply their acquired knowledge of the particle perspective to problems concerning structure-property reasoning. Students were more able to evaluate these problems on the less visible structural aspects such as type of particles or bonding. 
In the framed sorting task, the data showed a decrease in the FD score in both cohorts (Table 6). This means that after the demonstration-based lesson series students made fewer mistakes in sorting the problems into the appropriate predetermined categories. This applied specifically to the categories of metals and salts where students were found to make considerably fewer mistakes. This implies that the demonstration-based lesson series helped students to acquire structure models and, in their application, to solve problems. Students found the difference between bonds and lattices in the category of molecular compounds more difficult.

Table 6. Overview of the results of the framed sorting task of the SPR instrument.

\begin{tabular}{lcccccc}
\hline & & FD Score * & \multicolumn{3}{c}{ Percent Pairs } \\
& & & & Pre & Post & Difference \\
\hline Cohort 18-19 & Pre & 7.8 & Property & $21 \%$ & $18 \%$ & $-3 \%$ point \\
Cohort 19-20 & & 6.8 & & $20 \%$ & $19 \%$ & $-1 \%$ point \\
Cohort 18-19 & Post & 6.8 & Structure & $31 \%$ & $46 \%$ & $+15 \%$ point \\
Cohort 19-20 & & 6.4 & & $36 \%$ & $38 \%$ & $+2 \%$ point \\
\hline
\end{tabular}

* The FD score is defined by the number of cards that are placed in a group other than the ideal sort.

These predetermined categories helped the students to sort the problems into a structure level. This can also be seen in the higher \%P-structure score for the pretest and the posttest compared to the unframed sorting task (Table 6 for the framed sorting task and Table 7 for the unframed sorting task).

Table 7. Overview of the results of the unframed sorting task of the SPR instrument.

\begin{tabular}{clcccccc}
\hline & & \multicolumn{2}{c}{ Category Name is Referring to } & \multicolumn{3}{c}{ Percent Pairs (\%) } \\
& & Pre & Post & Difference & Pre & Post & Difference \\
\hline \multirow{2}{*}{ Property } & Cohort 18-19* & $74 \%$ & $66 \%$ & $-8 \%$ point & $70 \%$ & $61 \%$ & $-9 \%$ point \\
& Cohort 19-20\# & $84 \%$ & $58 \%$ & $-26 \%$ point & $82 \%$ & $71 \%$ & $-11 \%$ point \\
Structure & Cohort 18-19* & $3 \%$ & $12 \%$ & $+9 \%$ point & $8 \%$ & $13 \%$ & $+5 \%$ point \\
& Cohort 19-20 & $0 \%$ & $25 \%$ & $+25 \%$ point & $5 \%$ & $9 \%$ & $+4 \%$ point \\
\hline
\end{tabular}

* Cohort 18-19: Total number of categories produced in the pretest: 183; in the posttest: 189; ${ }^{\#}$ Cohort 19-20: Total number of categories produced in the pretest: 75 ; in the posttest: 79 .

\subsubsection{Unframed Sorting Task}

As shown in Table 7, students of both cohorts, mainly sorted on property aspects in both pretest and posttest. This finding confirmed the pre-university students' macroscopic orientation $[4,5]$. Examples of these category names were melting point, density and solubility. This macroscopic orientation was also depicted in the high \%P-property scores in the pretest and the posttest of both cohorts (Table 7).

At the same time, Table 7 shows a noteworthy increase in the mean \%P-structure scores in the posttest. In other words, students' sorts in the posttest were more similar to the ideal structure sort. This result shows an increase in students' proficiency in structure-property reasoning after the demonstration lessons. As the questions of the particle perspective were derived from the way an expert thinks [19], we could say that the students' evaluation of the problems became more similar to that of an expert.

\subsection{Case Study Sally}

To provide insight into how the demonstration-based lesson series impacted student learning, we present the case study of one student. Sally was a female student enrolled in the 19-20 cohort who was followed during the demonstration lesson on the topic of metals. She worked in a group with three other girls: Ryanna, Cathy and Fatima (fictive names). 


\subsubsection{During the Demonstration Lesson}

\section{FIRST STEP: PREDICT}

As the lesson started, Sally received an empty particle perspective and was asked by the teacher to complete the questions for the group of metals. The resulting perspective (see Figure 5 for Sally's first particle perspective, dotted underline) showed that her prior knowledge of the metals-properties and structure models—was quite comprehensive, meaning that her prior knowledge was good. Sally compared her personal particle perspective with that of others from her group in the subsequent group discussion, in which the four girls discussed the six questions for the metals and tried to formulate appropriate answers.

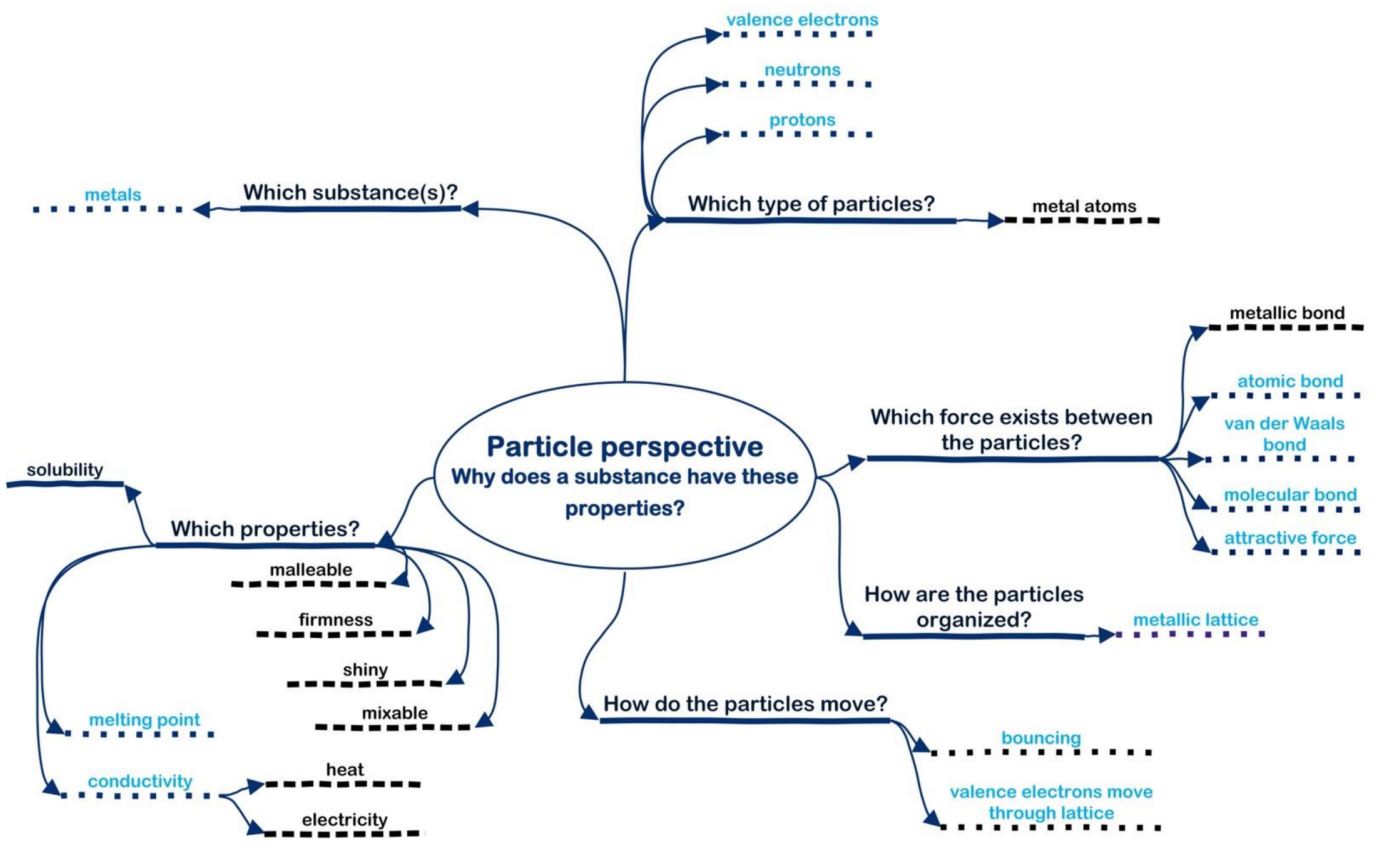

Figure 5. Sally's complete particle perspective before (underlined with dots) and additions after (underlined with dashes) the demonstration lesson on metals.

The audio recordings of Sally's group discussion revealed that her group started with the question "type of substance" (Figure 5). Next, they discussed "type of particles", and they named, among others, valance electrons (Figure 5) because of their role in conductivity. They also discussed "type of organization", how the valence electrons move through the lattice and the regularity of this lattice. They constantly switched between these two questions: "type of particles" and "type of organization" (Figure 5). Then they switched to the properties of metals and talked about the malleability, conductivity and mixability of metals (Figure 5, "which properties?"). Again, they discussed the ability of valence electrons to move through the lattice (Figure 5, "type of particles" and "how the particles are organized?"). At this point, they also named metallic particles. Then they listed all the bonds they knew, looking for a bond that fitted for the metals (Figure 5, "which force between the particles?").

The teacher then brought the predictions of the properties together in a class discussion. Together, the class named all the important properties of the metals, i.e., gray-colored, shiny after polishing, hard, malleable, solid at room temperature and able to conduct electricity and heat.

\section{SECOND STEP: OBSERVE}

In the demonstration, the teacher showed the properties of the metals. These were general aspects such as color, phase at room temperature, malleability, hardness and conductivity of electricity. There was a class discussion solely about the macroscopic properties of the metals. 


\section{THIRD STEP: EXPLAIN}

Sally's group started with the general model for particles, as shown in the second column of Table 8, and in their discussion, they immediately tried to take into account the conductivity of electricity, something they also named in their starting situation. They correctly suggested that valence electrons play a role in conductivity and that a neat lattice is needed for these electrons to move. They used the same size of circles for the particles in their drawing, showing that all the particles are equal. However, they paid no attention to the fact that a metal is malleable in their discussion of their first models (Table 8, column 2).

Table 8. Students' modeling of metals.

\begin{tabular}{|c|c|c|c|}
\hline First Model & 1st Adaptation & 2nd Adaptation & Final Model \\
\hline $\begin{array}{l}\text { T: Draw what a metal looks like at } \\
\text { particle level. } \\
\text { C: Something like a metal lattice, } \\
\text { right? } \\
\text { R: Nicely arranged lattice and } \\
\text { space for the valence electrons. } \\
\text { F: Are there neutrons, uh, is there } \\
\text { a neutron and a proton together? } \\
\text { C: It looks like... } \\
\text { R: Current and heat conductivity } \\
\text { has something to do with the } \\
\text { valence electrons. } \\
\text { S: So nicely arranged that it can } \\
\text { therefore easily pass through. } \\
\text { F: Valence electrons go just } \\
\text { through. } \\
\text { C: Nice circles, even, next to each } \\
\text { other, nicely arranged. } \\
\text { R: Do they have to be straight } \\
\text { next to each other? } \\
\text { F: I think so, so this way ... } \\
\text { S: And then like this, like building } \\
\text { blocks. Building blocks are also } \\
\text { arranged like this. } \\
\text { F: What was this called again? } \\
\text { S: A metallic lattice. } \\
\text { S: A lattice is nicely arranged. }\end{array}$ & $\begin{array}{l}\text { T: Now we have this model, can } \\
\text { you explain why a metal is } \\
\text { malleable? Draw! } \\
\text { R: Well, yes } \\
\text { S: Well, you can move those } \\
\text { things like this. } \\
\text { S: Look at this. This is what it } \\
\text { looks like. Look, I moved it. R: I } \\
\text { got that, too. }\end{array}$ & $\begin{array}{l}\text { T: Adapt your drawing so that it } \\
\text { is not malleable anymore. } \\
\text { S: With another substance in it, } \\
\text { other particles which are larger or } \\
\text { smaller. } \\
\text { F: Oh so. They are all of different } \\
\text { sizes now and then it can't deform } \\
\text { anymore. } \\
\text { R: If it is pure, it is easier to } \\
\text { deform than if it is an alloy. } \\
\text { S: An alloy can bend less easily } \\
\text { than a pure substance. }\end{array}$ & $\begin{array}{l}\text { T: Adapt your model so that it } \\
\text { explains the property conductivity. } \\
\text { C: That current that conducts } \\
\text { between all those things, doesn't } \\
\text { it? } \\
\text { R: With those valance electrons } \\
\text { that go everywhere in between. } \\
\text { F: Are those valence electrons } \\
\text { very small then? } \\
\text { C: I don't know. } \\
\text { R: They can move freely. }\end{array}$ \\
\hline
\end{tabular}

In the class discussion about the property malleability and the corresponding structure model, the teachers drew the common divisor of all the drawings she observed: equal round particles in a neat lattice. The teacher asked whether this structure model explained the property malleability. Sally mentioned that a row of particles can be moved without obstructions, and she showed it to her classmates in the drawing (Table 8, column 3).

After this adaptation of the structure model, the teacher asked the students to adapt the model to explain the hardness of an alloy such as steel (Table 8, column 4). Sally's group now recognized that differently sized particles are not able to move along easily and that malleability decreases, as Sally remarked: "With another substance in it, other particles which are larger or smaller" (Table 8, column 4). In a short class discussion, the adaptation of the structure model was discussed.

To explain the conductivity of electricity, the students had to adapt their structure model again. Sally's group discussed the role of the negatively charged valence electrons again, but the girls did not discuss the existence of the positively charged metal atoms. 
(Table 8, column 5). They correctly recognized that these electrons move freely through the lattice.

In the class discussion, the role of valence electrons was discussed. The students said that these electrons move freely: "they are playing tag". The teacher discussed the positively charged metal atom that appeared when a negatively charged electron moves through the lattice. The teacher asked the students to explain the conductivity of heat using this knowledge. Sally's group found it difficult to explain, but they discussed that the shaking or movement of a particle might play a role.

The adapted structure model as drawn in Table 8 (column 4) was now used to explain the high boiling point of metals. The teacher asked the class what was necessary on the structure level to obtain a high melting point on the property level. Sally suggested a strong bond between the particles. She also suggested that this strong bond originates in the attraction of positive and negative charges.

The last step of the lesson was the completion of the particle perspective again, now with all the concepts learned in this lesson (see Figure 5 for Sally's result; underlined with dashes). In a class discussion, the appropriate concepts for the metals were formulated for each question of the perspective.

4.2.2. Results of SPR Instrument of Sally's Group before and after the Demonstration Lesson Series

One week after the exam and five weeks after the last demonstration lesson, the students were asked to perform the SPR instrument as a posttest. Part of this test was the unframed mapping task, in which the students had to complete the questions of the particle perspective with the appropriate chemical concepts. The personal particle perspective showed the knowledge organization of the individual students. Sally's particle perspective (Figure 6) was very complete and comparable to the reference particle perspective (Figure 3). Some chemical concepts were missing from her particle perspective, mainly on the question "which type of particle?" (Figure 6). This concerns the concepts of metal and non-metal atoms and molecules.

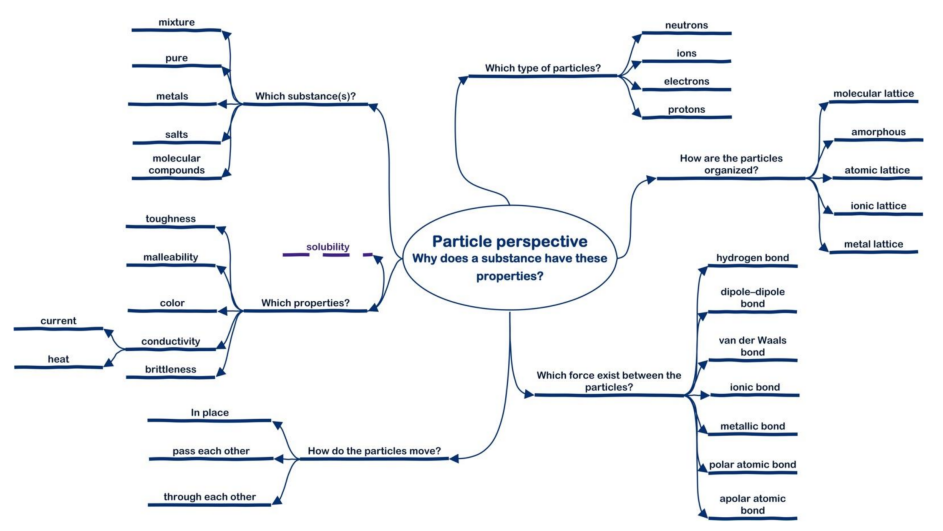

Figure 6. Posttest particle perspective of Sally, derived from the SPR instrument, unframed mapping task.

Sally's unframed sorting task showed a macroscopically orientated sort in the pretest. She used four categories: solubility, conductivity, melting point and hardness. These are the same categories that she also used in her posttest. Her \%P-property scores in the preand posttest were $72 \%$ and $84 \%$, respectively, meaning that her sorts were comparable with the ideal property sort, underlining her macroscopic orientation in these sorts. In the framed sorting task, she had a lower FD score in the posttest (decrease from 10 to 5). Her \%P-structure increased from $29 \%$ in the pretest to $44 \%$ in the posttest. With the predetermined categories in the framed sort, Sally was able to evaluate the problems on structural aspects after the demonstration series. This suggests that Sally increased her proficiency in structure-property reasoning. 


\section{Conclusions and Discussion}

This paper describes two design principles for demonstration-based lessons aiming to help students develop structure-property reasoning. The two design principles were: (1) adding a POE task to demonstrations to stimulate students' engagement and their modeling process and (2) scaffolding of the POE task with a domain-specific particle perspective $[18,19]$ in order to explicitly guide the modeling at the micro-level for students in the "explain" phase of the POE task. For the design of the lesson series, we explicitly used conventional demonstrations as a basis. The demonstration-based lesson series design with these two design principles was tested in two cohorts of upper pre-university students to investigate the effects of the two design principles on the level of their structure-property reasoning.

First, the results of the SPR instrument indicated that the demonstration series contributed to students' proficiency in structure-property reasoning. The unframed and framed mapping task of the SPR instrument showed that most students acquired and understood the chemical concepts needed for structure-property reasoning. In the framed mapping task, all students matched $97 \%$ of the given chemical concepts with the associated question of the particle perspective. In the posttest, the unframed mapping tasks were notably more comprehensive. Furthermore, the framed and unframed sorting tasks showed that students were better able to apply their acquired knowledge to problems concerning structure-property reasoning in the posttest. Students were also found to use more structure-property reasoning to evaluate and sort the types of problems that they were presented with in the posttest. The unframed and the framed sort in the posttest bore a greater resemblance to an ideal structure sort. Students also placed more cards in the "correct" category in the framed sort. In the unframed sorting task, the students used more category names referring to the structure level.

Considering design principle 1, adding the POE task, our data showed that the POE task engaged students in modeling of the structure level and, therefore, in acquiring structure level understanding. The qualitative data of Sally and her classmates (cohort 19-20) started with the general model for solids to explain the first property demonstrated. Sally and her classmates extended this model step-by-step by reviewing the model for the other demonstrated properties. Finally, they came to a model that explained all demonstrated properties of metals. During classroom reasoning, we could see that the properties demonstrated-solid at room temperature, malleability, hardness and conductivity of electricity-were used to create, extend and test their structure models. Moreover, Sally's particle perspective from the posttest (Figure 6) showed that she had acquired all the chemical concepts needed for proficient structure-property reasoning. In this study, we only followed the learning progression of one student. In further research, the learning progressions of a bigger group of students should be investigated.

Literature shows that one of the difficulties students experience with structureproperty reasoning is the connection between the macroscopic level of the properties and the micro-level where the structure models emerge. Due to their inexperience, students tend to start their reasoning from their macroscopic orientation [5,9]. By adding the "predict" step and the student-centered "explain" step, in which students actively construct the structure models and explain the predicted and observed properties, students had to make explicit connections between the two levels of representations. This might increase their proficiency in structure-property reasoning.

The qualitative data-Sally's drawings and her discussions with her classmates-also showed that the addition of the POE task to the demonstrations gave the students the opportunity to model the structure level themselves. After adapting her prior particle model of a solid (see the second row in Table 8), Sally formulated a structure model of metals that explained the demonstrated properties. It appears that the POE task facilitated students in the modeling process. Interestingly, the data also showed that this modeling process appeared to consist of several stages. Sally and her classmates did not merge all the shown properties into one comprehensive structure model in one take. Instead, the group first constructed a tentative model based on their prior knowledge and a general particle 
model and then extended this tentative model step-by-step into a more extensive model so that it could continue to explain new properties. In this way, Sally and her classmates explicitly commuted back and forth between properties and structure models. Such an iterative modeling process and back-and-forth thinking between properties and structure require the teacher to scaffold this well, e.g., by showing the properties in an order that supports an iterative modeling process.

Considering design principle 2 (scaffolding of the POE task with a domain-specific particle perspective) the SPR instrument-especially the unframed and framed mapping tasks-showed that students' particle perspective was more developed in the posttest. Developing the particle perspective also increased its value as a scaffold for the students. By obtaining the answers-the chemical concepts - to the question agenda, the students acquired the tools for structure-property reasoning. Furthermore, these chemical concepts were connected in functional coherence in the particle perspective.

Normally, students learn the chemical concepts, and as a next step, they apply these concepts in specific situations. In these demonstrations, students developed the chemical concepts in a context of structure-property reasoning. During the modeling process, the students had to work through the question agenda of the particle perspective several times, in an iterative process. Each time the particle perspective was extended, more options became available. These added concepts increased their proficiency in structure-property reasoning, and the students were able to question more complex and increasingly varied problems.

It is known from literature that one of the difficulties of structure-property reasoning that students experience is the invisibility of the structure level [5]. The structure level cannot be seen with the naked eye or through a microscope and models are needed to describe it. Because of this, structure-property relations become abstract and students are prone to misconceptions and experience various difficulties in solving problems $[5,6]$. In our study, the use of the particle perspective gave the students a scaffold to support the reasoning process by offering the concepts and the questions from the question agenda in coherence. Furthermore, the question agenda of the perspective gave the students insight into domain-specific reasoning. It enabled them to reason more like experts by questioning the problems with the aid of the question agenda. In sum, the particle perspective with its questions and the associated chemical concepts will increase the proficiency in students structure-property reasoning and will help them to solve problems with structure-property relations in the future.

The design of the demonstrations and the modeling process by the students worked best for metals and salts, as can be seen in the results of the framed and the unframed sorting tasks. These groups of compounds are well-defined groups with clear structureproperty relations and hardly any exceptions. The molecular compounds group was more difficult to demonstrate due to its complexity in terms of properties but also in terms of structure models: various types of bonds and lattices. This could probably be solved by dividing this group into several sub-groups of molecular compounds based on properties such as solubility and/or boiling points. In our demonstration, the problem was solved by reversing the design. Instead of asking them to model the structure level of molecular compounds, the students were asked to design appropriate demonstrations to help them find the answer to the questions of the particle perspective. This change of design was beneficial for teaching the students the particle perspective and thus for the explicit teaching of structure-property reasoning.

Both design principles helped to promote structure-property reasoning among students. However, these principles will only be used in day-to-day practices and on a wider scale if teachers estimate the principles to be practical. We know from the literature that teachers judge innovations to be practical based on three criteria: (1) the teaching practice needs to contain instrumental content so that teachers know how it will work in their setting; (2) the teaching practice needs to be congruent with teachers' goals and regular teaching practice; and (3) the teaching practice should be low-cost in terms of time and en- 
ergy that need to be invested [36,37]. In the present study, we used existing demonstrations as a starting point for redesign. We used the design principles to adapt these demonstrations. We expect that using existing elements (high instrumentality), a redesign close to teachers' existing teaching practices and materials already present (high congruency and cost-effectiveness) and the small change of the order of existing building blocks (Figure 1) amounts to high practicality for teachers. As demonstrations could be an important online teaching method in the present time of COVID-19, and as a weakness of online teaching is the lack of interaction with students, the addition of a POE task to demonstrations could increase the interactions with students making the online demonstration minds-on.

The combination of the two design principles together could be used in any situation in which students (from primary school to higher education) are asked to develop a model to explain phenomena. This is not only the case for science-related subjects but also, for example, in economics, social studies, geography or linguistics (as part of teaching a language). For the modeling of phenomena, the POE task could be used in the same manner each time, but for each domain, a different domain-specific perspective should be used. The perspective could act as a thinking frame for the students, and this might enhance the domain-specific way of thinking. Further research is needed for the development and implementation of both the particle perspective and the other domain-specific perspectives.

One lesson series to develop the particle perspective, associated chemical concepts and proficiency in using the question agenda of the particle perspective is clearly not enough Repetitive use of the question agenda and application of the chemical concepts in several assignments and tasks would be needed to mature structure-property reasoning. The lesson series described here could be the start of systematic use of the particle perspective for explicit teaching of structure-property reasoning. Further research could aim to develop strategies (tools) for teachers to design additional lessons using the particle perspective.

Author Contributions: Conceptualization, all authors; Methodology, all authors; Validation, M.J.d.O. and M.D.; Formal Analysis, M.-J.d.O. and M.D.; Investigation, M.-J.d.O.; Resources, M.J.d.O. and M.D.; Data Curation, M.-J.d.O. and M.D.; Writing—original draft preparation M.-J.d.O.; Writing-Review and Editing, all authors; Visualization, M.-J.d.O. and M.D.; Supervision, F.J. and L.J. All authors have read and agreed to the published version of the manuscript.

Funding: This research was supported by funding of the Dutch Ministry of Education, Culture and Science via the DUDOC program that focused on linking research and practice in educational settings.

Institutional Review Board Statement: The study was conducted according to the guidelines of the Declaration of Helsinki, and approved by the Ethics Committee of Leiden University Graduate School of Teaching (ICLON) (protocol code IREC_ICLON 2017-08 and date of approval 7th July 2017).

Informed Consent Statement: Informed consent was obtained from all subjects involved in the study.

Data Availability Statement: The data presented in this study are available on request from the corresponding author. The data are not publicly available due to privacy legislation.

Conflicts of Interest: The authors declare no conflict of interest. The funders had no role in the design of the study; in the collection, analyses or interpretation of data; in the writing of the manuscript or in the decision to publish the results.

\section{Appendix A. Teacher Demonstration Protocols}

In Tables A1-A3, the demonstration protocols for the demonstration experiments are provided. In these protocols, for each property, an accompanying demonstration is described. The structure model concept which could be modeled by the students is also indicated. These demonstrations fit in the "observe" phase in the POE task as described in "3.3. Overview of the Lesson Series".

For each demonstration, the properties of several substances of that group are demonstrated. The choice of substances depends on what is available at school to properly demonstrate the properties. The substances mentioned in the demonstration protocols are therefore only indicative. 
Table A1. Demonstration protocol for metals.

\begin{tabular}{|c|c|c|}
\hline Property & Demonstration Instructions & Structure Model \\
\hline Appearance & $\begin{array}{l}\text { The teacher shows different metals (sheets, rods, etc.), such as iron, } \\
\text { copper, lead, zinc. } \\
\text { The teacher polishes the metal plates and shows the results. }\end{array}$ & $\mathrm{n} / \mathrm{a}$ \\
\hline $\begin{array}{l}\text { Phase at room } \\
\text { temperature }\end{array}$ & The teacher shows the metal plates and asks about the phase. & Metallic lattice \\
\hline Strength & The teacher works the metal plates with a hammer. & Metallic lattice \\
\hline Malleability & $\begin{array}{l}\text { The teacher tries to bend the metal plates. } \\
\text { The teacher holds the metal lead }(\mathrm{mp}=327 \mathrm{~K}) \text { or zinc }(\mathrm{mp}=420 \mathrm{~K}) \text { in a }\end{array}$ & Metallic lattice \\
\hline Melting point & $\begin{array}{l}\text { blue flame. The metal becomes soft. Next, the teacher holds the metals } \\
\text { copper }(\mathrm{mp}=1083 \mathrm{~K}) \text { and /or iron }(\mathrm{mp}=1535 \mathrm{~K}) \text { in the flame. These } \\
\text { melting points are above the temperature }(1273 \mathrm{~K}) \text { of the blue flame and } \\
\text { will not soften. }\end{array}$ & Metallic bond \\
\hline Conductivity of electricity & $\begin{array}{l}\text { The teacher builds the setup to measure current conductivity: lamp, } \\
\text { voltage source, wires and, if necessary, adds a conductivity meter. The } \\
\text { teacher measures the current conductivity of various metals. } \\
\text { The teacher keeps a ribbon of magnesium in the flame. }\end{array}$ & Metallic lattice, metallic bond \\
\hline Behavior when heated & $\begin{array}{l}\text { The teacher sprinkles some metal powders (such as iron or magnesium) } \\
\text { through the flame. }\end{array}$ & $\mathrm{n} / \mathrm{a}$ \\
\hline
\end{tabular}

Table A2. Demonstration protocol for salts.

\begin{tabular}{|c|c|c|}
\hline Property & Demonstration & Structure Model \\
\hline Appearance & $\begin{array}{l}\text { The teacher shows different salts, such as sodium chloride, sodium } \\
\text { nitrate, iron (III) nitrate, copper sulfate. }\end{array}$ & $\mathrm{n} / \mathrm{a}$ \\
\hline Strength/malleability & $\begin{array}{l}\text { The teacher hits lump of salt with a hammer. At the school of the first } \\
\text { author, the assistant found an old bottle with big lumps of iron (III) } \\
\text { nitrate, which was suitable to hit it with a hammer. }\end{array}$ & $\begin{array}{l}\text { Lattice with } \\
\text { uneven particles }\end{array}$ \\
\hline Phase at room temperature & $\begin{array}{l}\text { The teacher shows different salts with attention to the phase at room } \\
\text { temperature. }\end{array}$ & Lattice with strong bonds \\
\hline Melting point & The teacher heats a salt such as sodium chloride and iron (III) nitrate. & Lattice with strong bonds \\
\hline Conductivity of electricity & $\begin{array}{l}\text { The teacher tests a solid salt, a liquid salt and a dissolved salt for } \\
\text { conductivity. }\end{array}$ & $\begin{array}{l}\text { The particles are charged and stuck } \\
\text { in a grid }\end{array}$ \\
\hline
\end{tabular}

Table A3. Demonstration protocol for molecular compounds.

\begin{tabular}{|c|c|c|}
\hline Property & Demonstration & Structure Model \\
\hline Appearance & $\begin{array}{l}\text { The teacher shows different molecular substances, such as sugar, } \\
\text { glucose, ethanol, water, methane (burning), oil. }\end{array}$ & $\mathrm{n} / \mathrm{a}$ \\
\hline Conductivity of electricity & $\begin{array}{l}\text { The teacher makes a sugar solution and an ethanol solution and tests } \\
\text { the current conduction. The teacher also tests the conductivity of water } \\
\text { and oil. }\end{array}$ & No charged particles: molecules \\
\hline Behavior when heated (sugar) & The teacher heats sugar until it caramelizes and burns. & $\begin{array}{l}\text { The molecules consist of atoms } \\
\text { The atomic bond is very strong }\end{array}$ \\
\hline Phase at room temperature & $\begin{array}{l}\text { The teacher shows liquid and gaseous molecular substances such as } \\
\text { water, } \mathrm{CO}_{2} \text { in soft drinks, methane gas, ethanol, acetone. }\end{array}$ & Weak bond between the molecules \\
\hline Boiling point & $\begin{array}{l}\text { The teacher boils water and ethanol and uses a sensor to measure the } \\
\text { temperature. } \\
\text { The students search the boiling points of the liquids and search for links. }\end{array}$ & $\begin{array}{l}\text { van der Waals bond and hydrogen } \\
\text { bond }\end{array}$ \\
\hline Solubility & $\begin{array}{l}\text { The teacher tries to dissolve various substances, such as sugar, oil in } \\
\text { water. Two groups emerge. }\end{array}$ & $\begin{array}{c}\text { van der Waals bond and hydrogen } \\
\text { bond }\end{array}$ \\
\hline Behavior when heated & The teacher heats up sugar and carbon in a rustling flame. & $\begin{array}{l}\text { Molecular lattice/molecular bond } \\
\text { vs. atomic lattice/atomic bond }\end{array}$ \\
\hline
\end{tabular}

\section{References}

1. Talanquer, V. Progressions in Reasoning about Structure-Property Relationships. Chem. Educ. Res. Pract. 2017, 19, 998-1009. [CrossRef]

2. National Research Council. A Framework for K-12 Science Education: Practices, Crosscutting Concepts, and Core Ideas; The National Academies Press: Washington, DC, USA, 2012; ISBN 978-0-309-21742-2.

3. Ottevanger, W.; Oorschot, F.; Spek, W.; Boerwinkel, D.J.; Eijkelhof, H.; de Vries, M.; van der Hoeven, M.; Kuiper, W. Kennisbasis Natuurwetenschappen En Technologie Voor de Onderbouw vo: Een Richtinggevend Leerplankader (Knowledge Base for Science and Technology for Year 1 to Year 3 of the Secondary Education: A Guiding Curriculum Framework); SLO: Enschede, The Netherlands, 2014. 
4. Johnstone, A.H. Why Is Science Difficult to Learn? Things Are Seldom What They Seem. J. Comput. Assist. Learn. 1991, 7, 75-83. [CrossRef]

5. Gabel, D.L. Improving Teaching and Learning through Chemistry Education Research: A Look to the Future. J. Chem. Educ. 1999, 76, 548-553. [CrossRef]

6. De Jong, O.; Taber, K.S. The Many Faces of High School Chemistry. In Handbook of Research on Science Education Volume II; Lederman, N.G., Abell, S.K., Eds.; Routledge Taylor and Francis group: New York, NY, USA, 2014; pp. 457-480. ISBN 9780415629553.

7. Talanquer, V. Chemistry Education: Ten Heuristics To Tame. J. Chem. Educ. 2014, 91, 1091-1097. [CrossRef]

8. Cooper, M.M.; Corley, L.M.; Underwood, S.M. An Investigation of College Chemistry Students' Understanding of StructureProperty Relationships. J. Res. Sci. Teach. 2013, 50, 699-721. [CrossRef]

9. Kozma, R.B.; Russell, J. Multimedia and Understanding: Expert and Novice Responses to Different Representations of Chemical Phenomena. J. Res. Sci. Teach. 1997, 34, 949-968. [CrossRef]

10. Stowe, R.L.; Herrington, D.G.; McKay, R.L.; Cooper, M.M. Adapting a Core-Idea Centered Undergraduate General Chemistry Curriculum for Use in High School. J. Chem. Educ. 2019, 96, 1318-1326. [CrossRef]

11. Kelly, R.M.; Jones, L.L. Investigating Students' Ability To Transfer Ideas Learned from Molecular Animations of the Dissolution Process. J. Chem. Educ. 2008, 85, 303-309. [CrossRef]

12. Ramsey, L.L.; Walczyk, J.; Deese, W.C.; Eddy, D. Using Demonstration Assessments to Improve Learning. J. Chem. Educ. 2000, 77, 1511-1516. [CrossRef]

13. Treagust, D.F. General instructional methods and strategies. In Handbook of Research on Science Education Volume II; Lederman, N.G., Abell, S.K., Eds.; Routledge Taylor and Francis group: New York, USA, 2014; pp. 373-391. ISBN 9780415629553.

14. Tsaparlis, G. Learning at the Macro Level: The Role of Practical Work. In Multiple Representations in Chemical Education; Gilbert, J.K., Treagust, D.F., Eds.; Springer: Dordrecht, The Netherlands, 2009; pp. 109-136. ISBN 978-1-4020-8871-1.

15. Hilario, J.S. The Use of Predict-Observe-Explain-Explore ( POEE ) as a New Teaching Strategy in General Chemistry-Laboratory. Int. J. Educ. Res. 2015, 3, 37-48.

16. Liew, C.-W.; Treagust, D.F. The Effectiveness of Predict-Observe-Explain Tasks in Diagnosing Students' Understanding of Science and in Identifying Their Levels of Achievement. Presented at the annual meeting of the American Educational Research Association, San Diego, CA, USA, 13-17 April 1998.

17. White, R.; Gunstone, R. Probing Understanding; The Falmer Press: London, UK, 1992.

18. Janssen, F.; Westbroek, H.; Landa, I.; van der Ploeg, B.; van Muijlwijk-Koezen, J.E. Perspectives for Teaching About How Science Works. In Nature of Science in Science Instruction: Rationales and Strategies; McComas, W.F., Ed.; Springer Nature Switzerland AG: Cham, Switzerland, 2020; pp. 253-270. ISBN 9783030572389.

19. Landa, I.; Westbroek, H.; Janssen, F.; van Muijlwijk, J.; Meeter, M. Scientific Perspectivism in Secondary-School Chemistry Education. Sci. Educ. 2020, 29, 1361-1388. [CrossRef]

20. Hodson, D. Learning Science, Learning about Science, Doing Science: Different Goals Demand Different Learning Methods. Int. J. Sci. Educ. 2014, 36, 2534-2553. [CrossRef]

21. Roth, W.-M.; Mcrobbie, C.J.; Lucas, K.B.; Boutonné, S. Why May Students Fail to Learn from Demonstrations? A Social Practice Perspective on Learning in Physics. J. Res. Sci. Teach. 1997, 34, 509-533. [CrossRef]

22. Crouch, C.; Fagen, A.P.; Callan, J.P.; Mazur, E. Classroom Demonstrations: Learning Tools or Entertainment? Am. J. Phys. 2004, 72, 835-838. [CrossRef]

23. Coştu, B.; Ayas, A.; Niaz, M. Investigating the Effectiveness of a POE-Based Teaching Activity on Students' Understanding of Condensation. Instr. Sci. 2011, 40, 47-67. [CrossRef]

24. Kala, N.; Yaman, F.; Ayas, A. The Effectiveness Of Predict-Observe-Explain Technique In Probing Students' Understanding About Acid-Base Chemistry: A Case For The Concepts Of Ph, Poh, And Strength. Int. J. Sci. Math. Educ. 2013, 11, 555-574. [CrossRef]

25. Karamustafaoğlu, S.; Mamlok-Naaman, R. Understanding Electrochemistry Concepts Using the Predict-Observe-Explain Strategy. EURASIA J. Math. Sci. Technol. Educ. 2015, 11, 923-936. [CrossRef]

26. Kearney, M.; Treagust, D.F.; Yeo, S.; Zadnik, M.G. Student and Teacher Perceptions of the Use of Multimedia Supported Predict-Observe-Explain Tasks to Probe Understanding. Res. Sci. Educ. 2001, 31, 589-615. [CrossRef]

27. Smith, K.C.; Edionwe, E.; Michel, B. Conductimetric Titrations: A Predict-Observe-Explain Activity for General Chemistry. J. Chem. Educ. 2010, 87, 1217-1221. [CrossRef]

28. Yaman, F.; Ayas, A. Assessing Changes in High School Students' Conceptual Understanding through Concept Maps before and after the Computer-Based Predict-Observe-Explain (CB-POE) Tasks on Acid-Base Chemistry at the Secondary Level. Chem. Educ. Res. Pract. 2015, 16, 843-855. [CrossRef]

29. Zakiyah, I.; Widodo, W.; Tukiran, T. Implementation of Predict-Observe-Explain (POE) Strategy to Reduce Misconception in Thermochemistry. Int. J. Educ. Vocat. Stud. 2019, 1, 754-759. [CrossRef]

30. Zakiyah, I.; Widodo, W.; Tukiran, T. Profile of Student's Conception in Implementation of Predict-Observe-Explain (POE) Strategy on Thermochemistry Concept. J. Phys. Conf. Ser. 2020, 1567. [CrossRef]

31. Kibirige, I.; Osodo, J.; Tlala, K.M. The Effect of Predict-Observe-Explain Strategy on Learners' Misconceptions about Dissolved Salts. Mediterr. J. Soc. Sci. 2014, 5, 300-310. [CrossRef]

32. Mattox, A.C.; Reisner, B.A.; Rickey, D. What Happens When Chemical Compounds Are Added to Water? An Introduction to the Model-Observe-Reflect-Explain (MORE) Thinking Frame. J. Chem. Educ. 2006, 83, 622. [CrossRef] 
33. Tien, L.T.; Teichert, M.A.; Rickey, D. Effectiveness of a MORE Laboratory Module in Prompting Students To Revise Their Molecular-Level Ideas about Solutions. J. Chem. Educ. 2007, 84, 175-181. [CrossRef]

34. van de Pol, J.; Volman, M.; Beishuizen, J. Scaffolding in Teacher-Student Interaction: A Decade of Research. Educ. Psychol. Rev. 2010, 22, 271-296. [CrossRef]

35. den Otter, M.J.; Juurlink, L.B.F.; Janssen, F.J.J.M. How to Assess Students' Structure-Property Reasoning? Chem. Educ. Res. Pract. 2021. Submitted.

36. Janssen, F.J.J.M.; Westbroek, H.B.; Doyle, W. The Practical Turn in Teacher Education. J. Teach. Educ. 2014, 65, 195-206. [CrossRef]

37. Doyle, W.; Ponder, G.A. The Practicality Ethic in Teacher Decision-Making. Interchange 1977, 8, 1-12. [CrossRef] 